\title{
Power Control and Rate Management for Wireless Multimedia CDMA Systems
}

\author{
Chi Wan Sung, Member, IEEE, and Wing Shing Wong, Senior Member, IEEE
}

\begin{abstract}
We consider a wireless multimedia code-division multiple-access system, in which the terminals transmit at different rates. We formulate the problem as a constrained optimization problem, with the objective of maximizing the total effective rate. An optimal power control strategy is derived. When the scale of the system is large, the optimal solution takes a simple form, which is easy to be applied practically. Furthermore, our basic model can be extended to include delay-sensitive traffic.
\end{abstract}

Index Terms-Multimedia CDMA, multirate CDMA, power control.

\section{INTRODUCTION}

$\mathbf{S}$ PEECH transmission is the main service supported by the first two generations of mobile communication systems. However, future systems should be able to handle a wide variety of different services with bit rate requirement ranging from a few kilobits/s to as much as $2 \mathrm{Mb} / \mathrm{s}$. In this paper, we consider using code-division multiple access (CDMA) as the access method. There are many ways to design a CDMA system to support multirate services [8]. One way to do so is to spread all signals, independent of the bit rate, to the same bandwidth [1], [5]. This is done by keeping the chip rate constant. Users transmitting at low bit rate thus have a high processing gain. This allows those users to transmit at a lower power. Therefore, the conventional constant received power scheme is not appropriate in such a multirate system. A more sophisticated power control scheme is needed to achieve diverse quality of service (QoS) and rate requirement. One objective of this paper is to explore the relationship between the data rate, the QoS, and the transmit power.

In the literature, the power control problem is usually formulated as an optimization problem which minimizes the transmit power subject to minimum signal-to-interference (SIR) requirement [3], [15], [16], [21]. This approach is very suitable for handling voice traffic. For some other multimedia applications, however, different objectives may be more appropriate. Thus, different power control schemes have been proposed. For instance, in [20], schemes based on the equal signal strength rule

Paper approved by E. S. Sousa, the Editor for CDMA Systems of the IEEE Communications Society. Manuscript received July 27, 1998; revised May 30 2000. This work was supported by the Hong Kong Research Grants Council. This paper was presented in part at the INFOCOM'99, New York, NY, March 1999.

C. W. Sung is with the Department of Computer Science, City University of Hong Kong, Kowloon, Hong Kong (e-mail: cwsung@cs.cityu.edu.hk).

W. S. Wong is with the Department of Information Engineering, Chinese University of Hong Kong, Shatin N.T., Hong Kong (e-mail: wswong@ie.cuhk.edu.hk).

Publisher Item Identifier S 0090-6778(01)05775-0. and the equal error probability rule are used. The objective is to guarantee the minimum required voice quality and reserve the highest possible system capacity to data users. However, their approach considers only two traffic types and has no natural generalization to multimedia traffic. In [22], the power levels are obtained via dynamic programming. In [19], linear programming and nonlinear programming are used. Since a lot of computation is needed in those approaches, it may not be suitable to apply them to a practical system, in which the power levels must be obtained in real time so as to adapt to the changing environment and traffic load. In [5], simple rules based on heuristic reasoning are given. A power control algorithm for heterogeneous sources is proposed in [7].

While most of the existing work in this area has been done with respect to a specific transmitter and receiver structure, an information theoretic treatment which characterizes the capacity region of a multiaccess fading channel can be found in [17]. The optimal resource allocation scheme which attains the points on the boundary of the capacity region was obtained. However, the resource allocation scheme is very difficult to implement practically. For instance, for equal-rate sources, the optimal scheme allows only one user to access the channel at any given time. The one with the largest instantaneous link gain can transmit while others should remain silent. This is difficult to achieve, especially if the number of users is large and the channel changes rapidly. In our work, we take a different approach. We ask the following question: how should we set the SIR for delay tolerant sources? Such sources can potentially benefit from coding, which is obtained at the cost of delay. We assume that the various sources differ only in their data rates. Our goal is to maximize the sum of effective rate of all the sources. Once the optimal SIR values have been determined, distributed power control algorithms such as those proposed in [3] and [15] can be used to maintain the target SIR even in a fading channel.

The main theme of this paper is to find an efficient way to allocate resources for delay-tolerant sources. However, our model can be easily extended to include delay-sensitive sources such as voice and video. For these sources, we assume that a desired SIR must be met [3], [15], [21]. We show that the coexistence of delay-tolerant and delay-sensitive sources in a system has no effect on our main result as the problem can be easily decoupled. An information-theoretic treatment on delay-sensitive sources can be found in [4].

This paper is organized as follows. In Section II, the system model is described and the problem is formulated. The power control scheme is derived in Section III. Application to a largescale system is considered in Section IV. An extension to the 
basic model is given in Section V. In Section VI, we present a numerical study. Conclusion will be made in the last section.

\section{SySTEM MOdEL AND PROBLEM FormULATION}

We consider a single-cell CDMA system. We let $N$ be the number of terminals in the system. In general, the data rate of each terminal can be different. We denote the raw data rate of terminal $i$ by $R_{i}$. To support multirate services, we consider a variable spreading gain CDMA system. In such a system, the chip rate $R_{c}$ of each terminal is the same. Thus, the signals of all users are spread to the same bandwidth $W$. Since each terminal has different transmission rate, the spreading gain is different for each of them. In this paper, we consider the uplink channel only. In mobile communications, the propagation loss is due to effects including distance loss and shadow fading. These effects are captured by the link gains. We use the notation $G_{i}$ to represent the link gain between terminal $i$ and the base station. We let $\eta$ be the receiver noise power and $P_{i}$ be the transmit power of terminal $i$. Thus, the SIR $\Gamma_{i}$ can be written as

$$
\begin{aligned}
\Gamma_{i} & =\frac{G_{i} P_{i}}{\sum_{j \neq i} G_{j} P_{j}+\eta} \\
& =\frac{Q_{i}}{\sum_{j \neq i} Q_{j}+\eta}
\end{aligned}
$$

where $Q_{i}=G_{i} P_{i}$ is the power of terminal $i$ received at the base station. We define $f$ as the highest rate in bits per channel use at which information can be sent with arbitrarily low probability of error. In information theoretic terminology, $f$ is the capacity of a discrete-time channel. In digital communications, it is well known that the raw bit-error rate (BER) is a function of $E_{b} / J_{0}$ [10]. We assume that $f$ is also a function of $E_{b} / J_{0}$, which can be related to the SIR by the following formula [6]:

$$
\left(\frac{E_{b}}{J_{0}}\right)_{i}=\frac{\Gamma_{i} W}{R_{i}} .
$$

In our model, terminal $i$ uses the channel $R_{i}$ times per unit time. Thus, the information rate through the channel is given by

$$
R_{i} f\left(\frac{\Gamma_{i} W}{R_{i}}\right) .
$$

We call it the effective data rate of user $i$. The total effective rate of all the data terminals $R_{T}$ is simply the sum of them

$$
R_{T}=\sum_{i=1}^{N} R_{i} f\left(\frac{\Gamma_{i} W}{R_{i}}\right)
$$

Our objective is to maximize $R_{T}$ by determining an optimal power vector $\mathbf{P}=\left(P_{1}, P_{2}, \ldots, P_{N}\right)$. Since the SIR depends only on the received power vector, we can treat $\mathbf{Q}=\left(Q_{1}, Q_{2}, \ldots, Q_{N}\right)$ as the independent variables.

For illustration, we consider the following model. We assume a binary symmetric channel (BSC) for each user. The crossover probability is denoted by $p$. The channel capacity is given by [2]

$$
C=1+p \log p+(1-p) \log (1-p) .
$$

The crossover probability $p$ is a function of $E_{b} / J_{0}$. The explicit form depends on the modulation scheme. We use the binary phase-shift keying (BPSK) and the differential phase-shift keying (DPSK) as examples. Hence, the function $f(x)$ can be expressed as follows:

$$
f(x)=1+p(x) \log p(x)+(1-p(x)) \log (1-p(x))
$$

where

$$
p(x)= \begin{cases}\frac{1}{2} \operatorname{erfc}(\sqrt{x}), & \text { for BPSK } \\ \frac{1}{2} \exp (-x), & \text { for DPSK. }\end{cases}
$$

Our results derived in this paper do not necessarily confine to this particular model. It can be applied to other models as long where $f(x)$ satisfies certain conditions. Before we describe the conditions, we first introduce the notion $\mathcal{P}$.

For a function $g(x)$, we define $\mathcal{P}(g(x), c)$ as the property that there exists $c>0$ such that

$$
g(x) \begin{cases}>0, & \text { for } 0<x<c \\ =0, & \text { for } x=c \\ <0, & \text { for } x>c .\end{cases}
$$

Now we describe the following conditions imposed on $f(x)$.

1) $f:[0, \infty) \rightarrow[0,1)$ is continuous.

2) $f(0)=0$ and $\lim _{x \rightarrow \infty} f(x)=1$.

3) $f^{\prime}:(0, \infty) \rightarrow\left(0, f_{\max }^{\prime}\right)$, for some finite constant $f_{\max }^{\prime}>$ 0 .

4) For any $x \in(0, \infty)$, the first three derivatives of $f$ exists and the third derivative is continuous.

5) $f^{\prime \prime}:(0, \infty) \rightarrow\left(f_{\min }^{\prime \prime}, f_{\max }^{\prime \prime}\right)$, for some constants $f_{\min }^{\prime \prime}$ and $f_{\max }^{\prime \prime}$ where $f_{\min }^{\prime \prime}<f_{\max }^{\prime \prime}$.

6) Either $f^{\prime \prime}(x)<0$ or $\mathcal{P}\left(f^{\prime \prime}(x), \beta_{0}\right)$.

7) $f^{\prime}(x)=o\left(x^{-2}\right)(x \rightarrow \infty)$.

8) Define $h(x)=x^{2} f^{\prime}(x)$. We have $\mathcal{P}\left(h^{\prime}(x), x_{0}\right)$ for some $x_{0}$.

Totally there are eight conditions, which may look clumsy at first sight. However, these conditions are not too restrictive. Condition 1 requires the input alphabet of the channel to be binary such that at most one bit can pass through reliably per channel use. Conditions 2 and 3 require $f$ to be an increasing function of $E_{b} / J_{0}$ and when $E_{b} / J_{0}$ becomes extremely large, every bit can pass through the channel reliably. Conditions 4 and 5 are mild conditions which are satisfied by many functions arising in engineering applications. Thus, what matter most are the last three conditions. Condition 6 requires $f$ to be either concave, or convex for small $x$ and concave for large $x$. Condition 7 is another way of saying that $\lim _{x \rightarrow \infty} h(x)=0$. Condition 8 is equivalent to $\mathcal{P}\left(x f^{\prime \prime}(x)+2 f^{\prime}(x), x_{0}\right)$. Conditions 3 and 8 together imply that $f^{\prime \prime}(x)<0$ for $x>x_{0}$.

In Figs. 1 and 2, we plot $f(x)$ and its derivatives for the BSC model with BPSK and DPSK modulation, respectively. The formulas of the derivatives are stated in Appendixs A and B for references. From the figures, it can be seen that $f(x)$ satisfies the required conditions in both cases. Note that in the BPSK case, $f^{\prime \prime}(x)<0$ for all $x$, while in the DPSK case, we have $\mathcal{P}\left(f^{\prime \prime}(x), \beta_{0}\right)$ where $\beta_{0}=0.831$. Condition 8 is also satisfied 

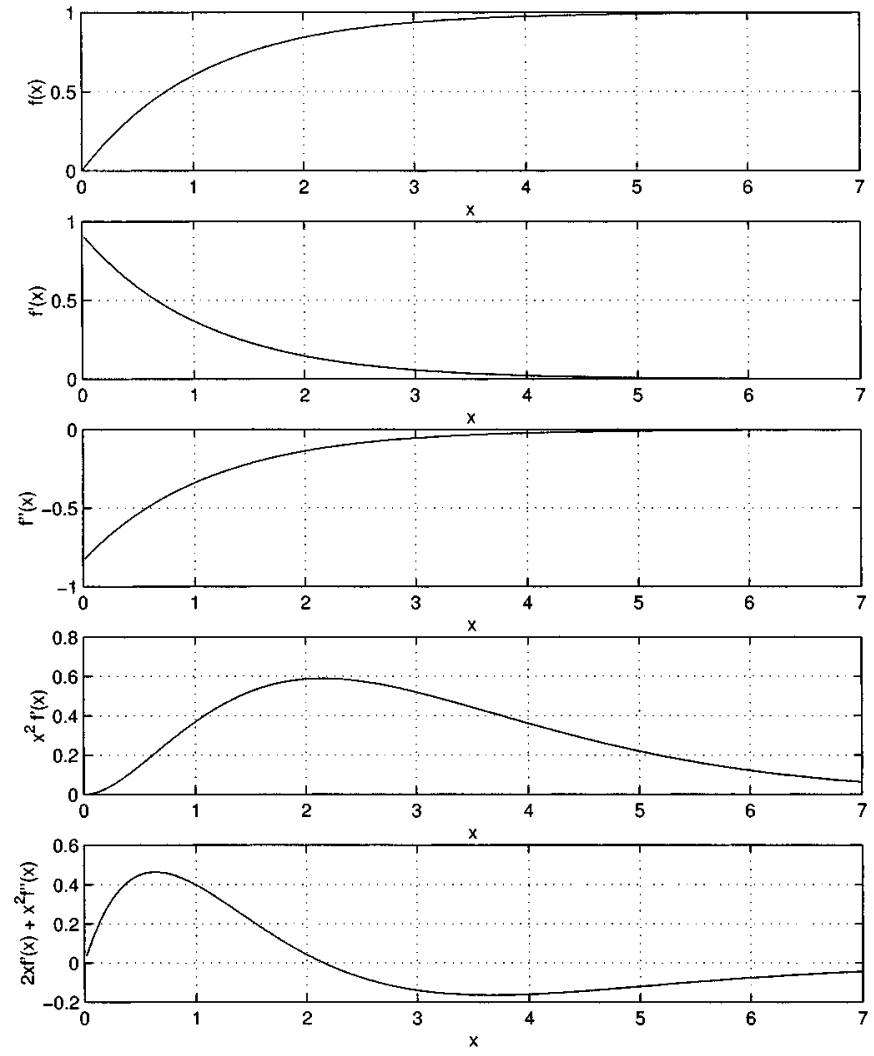

Fig. 1. $f(x)$ and its derivatives for BSC channel with BPSK modulation.

for both modulation scheme. The value of $x_{0}$ is equal to 2.154 and 2.835 for BPSK and DPSK, respectively.

These two explicit forms of $f$ will be used in our numerical sections. However, our analytical result only requires $f$ to satisfy the required conditions. It can be verified that some common functions such as $1-\exp (-x)$ and $\exp (-1 / x)$ also satisfy the conditions.

\section{POWER CONTROL SCHEME}

In our model, the effect of receiver noise $\eta$ is included. If $\eta>0$, it is easy to see that scaling up any power vector $\mathbf{Q}$ will improve the SIR of all users. As a result, no optimal solution can be found. In practice, however, dynamic range limitations at the base station receiver require that the total received power be limited [13], [18]. A rule of thumb adopted by [13] and [18] is to restrict the total received power to within $10 \mathrm{~dB}$ above the background noise level $\eta$. Therefore, we impose the following equality constraint:

$$
\sum_{i=1}^{N} Q_{i}=Q_{T}
$$

To simplify the computational complexity of the problem, we employ the following coordinate transformation:

$$
1+\Gamma_{i}=\frac{\sum_{j} Q_{j}+\eta}{\sum_{j \neq i} Q_{j}+\eta}
$$
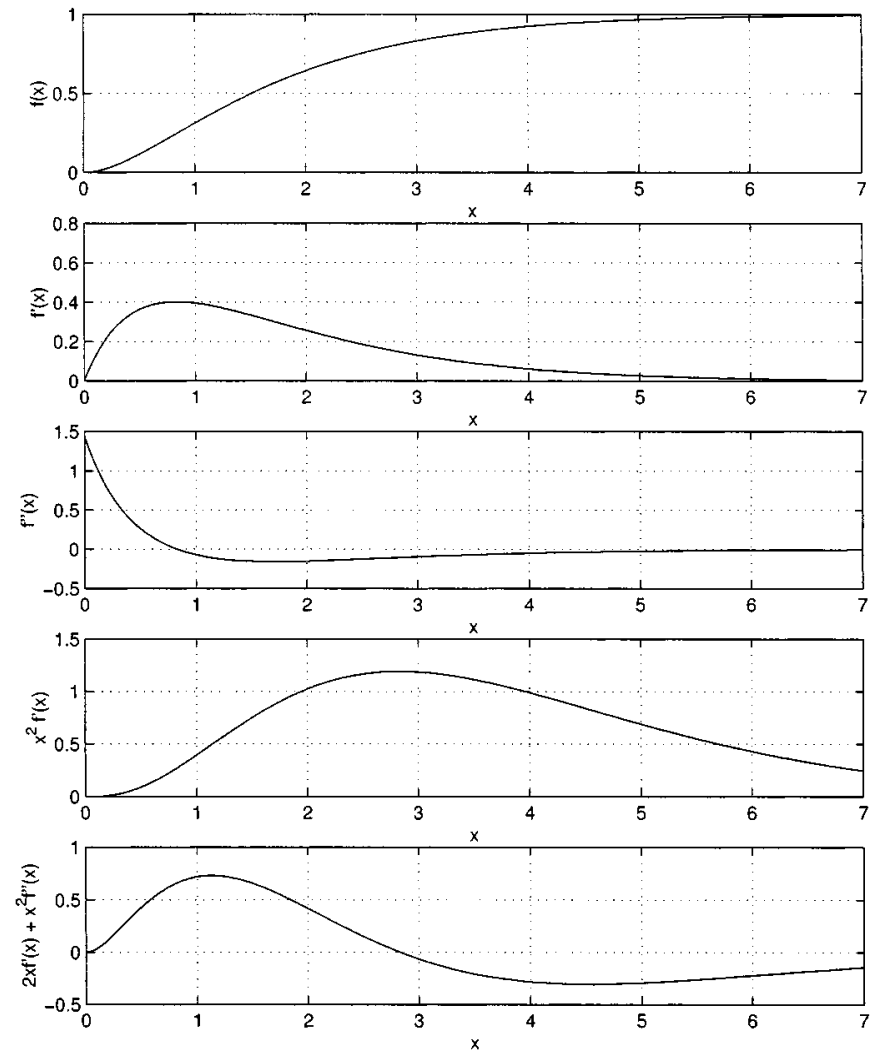

Fig. 2. $f(x)$ and its derivatives for BSC channel with DPSK modulation.

$$
\begin{aligned}
& =\frac{Q_{T}+\eta}{Q_{T}+\eta-Q_{i}} \\
\frac{1}{1+\Gamma_{i}} & =1-\frac{Q_{i}}{Q_{T}+\eta} .
\end{aligned}
$$

There is a one-to-one mapping between nonnegative $\Gamma_{i}$ and $Q_{i}$. The constant received power constraint becomes

$$
\sum_{i=1}^{N} \frac{1}{1+\Gamma_{i}}=N-\frac{Q_{T}}{Q_{T}+\eta} .
$$

To solve our problem, we use the method of Lagrange multiplier. We define the Lagrangian

$$
L=\sum_{i=1}^{N} R_{i} f\left(\frac{\Gamma_{i} W}{R_{i}}\right)+\lambda\left(\sum_{i=1}^{N} \frac{1}{1+\Gamma_{i}}-K\right)
$$

where $K=N-\left(Q_{T} /\left(Q_{T}+\eta\right)\right)$ is a constant.

In order for $R_{T}$ to attain an extremum, the following $N+1$ equations must be satisfied:

$$
\begin{aligned}
& \frac{\partial L}{\partial \Gamma_{i}}=W f^{\prime}\left(\frac{\Gamma_{i} W}{R_{i}}\right)-\frac{\lambda}{\left(\Gamma_{i}+1\right)^{2}}=0 \\
& \quad \text { for } i=1,2, \ldots, N \\
& \frac{\partial L}{\partial \lambda}=\sum_{i=1}^{N} \frac{1}{1+\Gamma_{i}}-K=0 .
\end{aligned}
$$


This system of $N+1$ equations enables us to solve for the $N+1$ unknowns $\Gamma_{1}, \Gamma_{2}, \ldots \Gamma_{N}$ and $\lambda$. We call (15) the key equation. It can also be expressed as follows:

$$
W k_{i}\left(x_{i}\right)=\lambda
$$

where $x_{i}=\Gamma_{i} W / R_{i}$, and

$$
k_{i}\left(x_{i}\right)=\left(1+\frac{x_{i} R_{i}}{W}\right)^{2} f^{\prime}\left(x_{i}\right) .
$$

If a solution exists, we denote it by $x_{i}^{*}$. The corresponding value of $\Gamma_{i}$ is denoted by $\Gamma_{i}^{*}$.

The nature of the stationary point is governed by the second derivative

$$
\frac{\partial^{2} L}{\partial \Gamma_{i}^{2}}=\frac{W^{2}}{R_{i}} f^{\prime \prime}\left(\frac{\Gamma_{i} W}{R_{i}}\right)+\frac{2 \lambda}{\left(\Gamma_{i}+1\right)^{3}}
$$

and

$$
\frac{\partial^{2} L}{\partial \Gamma_{i} \partial \Gamma_{j}}=0, \quad i \neq j .
$$

Due to (20), the sufficient condition for the stationary point to be a local maximum becomes [11]

$$
\left.\frac{\partial^{2} L}{\partial \Gamma_{i}^{2}}\right|_{\Gamma^{*}, \lambda^{*}}<0, \quad \text { for } i=1,2, \ldots, N
$$

and for it to be a local minimum, the sufficient condition is

$$
\left.\frac{\partial^{2} L}{\partial \Gamma_{i}^{2}}\right|_{\Gamma^{*}, \lambda^{*}}>0, \quad \text { for } i=1,2, \ldots, N .
$$

If we substitute (15) into (19), we have

$$
\begin{aligned}
\left.\frac{\partial^{2} L}{\partial \Gamma_{i}^{2}}\right|_{\Gamma^{*}, \lambda^{*}} & =\frac{W^{2}}{R_{i}} f^{\prime \prime}\left(\frac{\Gamma_{i}^{*} W}{R_{i}}\right)+\frac{2 W}{\Gamma_{i}^{*}+1} f^{\prime}\left(\frac{\Gamma_{i}^{*} W}{R_{i}}\right) \\
& =W\left[\frac{W}{R_{i}} f^{\prime \prime}\left(x_{i}^{*}\right)+\frac{2}{\Gamma_{i}^{*}+1} f^{\prime}\left(x_{i}^{*}\right)\right] \\
& =\frac{W^{2}}{R_{i}\left(1+R_{i} x_{i}^{*} / W\right)^{2}} k_{i}^{\prime}\left(x_{i}^{*}\right)
\end{aligned}
$$

where

$$
k_{i}^{\prime}\left(x_{i}\right)=\frac{R_{i}}{W}\left(1+\frac{x_{i} R}{W}\right)\left[2 f^{\prime}\left(x_{i}\right)+\left(\frac{W}{R_{i}}+x_{i}\right) f^{\prime \prime}\left(x_{i}\right)\right]
$$

is obtained by differentiating $k_{i}\left(x_{i}\right)$.

This equation will be used in sequel to determine whether a stationary point yields a maximum or a minimum.

Now we come to the point to show the existence of $\boldsymbol{\Gamma}^{*}$. If it exists and attains a global maximum, then the optimality can be achieved by allowing all users to transmit simultaneously. We call such a solution a harmonious schedule. Otherwise, if the solution precludes some users from transmitting, we call it a dominated schedule. We summarize our results in a series of lemmas, theorems and corollaries.
Lemma 1: Given any $\lambda$ where $0<\lambda \leq W f^{\prime}\left(x_{0}\right)$, if $W$ is large enough, then for all $i$, there exists a unique $x_{i}^{*}$ at which

$$
\frac{\partial L}{\partial \Gamma_{i}}=0
$$

and

$$
\frac{\partial^{2} L}{\partial \Gamma_{i}^{2}}<0 .
$$

Furthermore, $x_{i}^{*}$ is a strictly decreasing function of $\lambda$, and we have $x_{i}^{*} \geq x_{0}$.

Proof: By Lemma 3, which is shown in Appendix B, for sufficiently large $W$, if $f^{\prime \prime}(x)<0$ for all $x$, we have $k_{i}^{\prime}\left(x_{i}\right)<0$ for all $i$ and all $x_{i}>0$. Otherwise, if $\mathcal{P}\left(f^{\prime \prime}(x), \beta_{0}\right)$, then we have $\mathcal{P}\left(k_{i}^{\prime}\left(x_{i}\right), \alpha_{i}\right)$, where $\alpha_{i} \in\left(\beta_{0}, x_{0}\right)$ for all $i$.

In the first case where $\alpha_{i}$ does not exist, we define $\alpha_{i}=0$ for all $i$. Then in both cases, $k_{i}\left(x_{i}\right)$ is a strictly decreasing function of $x_{i}$ for $x_{i} \in\left(\alpha_{i}, \infty\right)$.

It is easy to see that

$$
W k_{i}\left(\alpha_{i}\right)>W k_{i}\left(x_{0}\right)>W f^{\prime}\left(x_{0}\right)
$$

for all $i$.

Condition 7 implies that

$$
W \lim _{x_{i} \rightarrow \infty} k_{i}\left(x_{i}\right)=0 .
$$

Hence, given any $\lambda$ where $0<\lambda \leq W f^{\prime}\left(x_{0}\right)$, we can find an unique $x_{i}^{*} \in\left(\alpha_{i}, \infty\right)$ such that

$$
W k_{i}\left(x_{i}^{*}\right)=\lambda .
$$

Furthermore, $x_{i}^{*}$ is a strictly decreasing function of $\lambda$.

If $\alpha_{i}>0$, assume that there exists $x_{i}^{\prime}<\alpha_{i}$ such that

$$
W k_{i}\left(x_{i}^{\prime}\right)=\lambda .
$$

By (25) and the property that $\mathcal{P}\left(k_{i}^{\prime}\left(x_{i}\right), \alpha_{i}\right)$, we have

$$
\begin{aligned}
& \left.\frac{\partial^{2} L}{\partial \Gamma_{i}^{2}}\right|_{x_{i}^{*}}<0 \\
& \left.\frac{\partial^{2} L}{\partial \Gamma_{i}^{2}}\right|_{x_{i}^{\prime}}>0 .
\end{aligned}
$$

Thus, the claim follows.

Lemma 2: If $W$ is large enough, $R_{T}$ has a unique extremum at $\mathrm{x}^{*}$, where $x_{i}^{*}>x_{0}$ for all $i$. Furthermore, it is a local maximum.

Proof: We have already shown that $\left.\left(\partial^{2} L / \partial \Gamma_{i}^{2}\right)\right|_{x_{i}^{*}}<0$ for all $i$. However, we need to ensure that the constraint

$$
\sum_{i} \frac{1}{1+\Gamma_{i}^{*}}=K
$$

is met.

If the above summation is greater than $K$, then we decrease $\lambda^{*}$. The effect is that $x_{i}^{*}$ will increase and in turn $\Gamma_{i}^{*}$ will also increase. If $\lambda^{*} \rightarrow 0$, then $\Gamma_{i}^{*} \rightarrow \infty$ and $1 /\left(1+\Gamma_{i}^{*}\right) \rightarrow 0<K$. Hence, there exists a $\lambda^{*}$ such that the constraint is satisfied. 
Now we consider the second case. If the summation is less than $K$, we may not have a solution. Suppose we increase $W$. The value of $\alpha_{i}$, which is defined in the Proof of Lemma 1, may change, but the strictly decreasing property remains the same. Assume that we increase $\lambda^{*}$ proportionally such that $\lambda^{*} / W=$ $f^{\prime}\left(x_{0}\right)$. Then $x_{i}^{*}$ is the root of $k_{i}\left(x_{i}\right)=f^{\prime}\left(x_{0}\right)$. Due to the increase in $W$, the curve $k_{i}\left(x_{i}\right)$ will move down. As a result, $x_{i}^{*}$ will decrease. Thus, when $W \rightarrow \infty, \Gamma_{i}^{*}=\left(x_{i}^{*} R_{i} / W\right) \rightarrow 0$. In other words, we have

$$
\lim _{W \rightarrow \infty} \sum_{i} \frac{1}{1+\Gamma_{i}^{*}}=N>K .
$$

Hence, if $W$ is sufficiently large, we must have

$$
\sum_{i} \frac{1}{1+\Gamma_{i}^{*}}>K
$$

Then we can decrease $\lambda^{*}$ until the equality constraint is met.

Note that in $\left\{\mathbf{x} \mid x_{i}>\alpha_{i} \forall i\right\}$, the stationary point $\mathrm{x}^{*}$ is unique. By Lemma 1, it attains a local maximum.

Now we consider the case where $\alpha_{i}>0$ for some $i$. If there is another stationary point $\mathrm{x}^{\prime}\left(\neq \mathrm{x}^{*}\right.$ in $\left\{\mathrm{x} \mid x_{i}>0 \forall i\right\}$, there must be some $x_{i}^{\prime}<\alpha_{i}$. Therefore, we have

$$
\left.\frac{\partial^{2} L}{\partial \Gamma_{i}^{2}}\right|_{\mathbf{x}_{\mathbf{i}}^{\prime *}, \lambda^{*}}>0 \text {. }
$$

Due to the equality constraint, it is impossible that $x_{i}^{\prime}<x_{i}^{*}$ for all $i$. Therefore, $\mathrm{x}^{\prime}$ does not yield a local minimum. It can only yield a saddle point. Hence, $R_{T}$ possesses only one extremum and the claim follows.

Finally, we show that this local maximum is in fact a global maximum.

Theorem 1: For the multirate power control problem with $N$ users, if the bandwidth is large enough, then the capacity optimization problem has a harmonious solution at which a strong ${ }^{1}$ global maximum can be attained.

Proof: By Lemma 2, $R_{T}$ has only one extremum. Hence the global maximum can be attained either at the boundary or at $\mathrm{x}^{*}$. At the boundary, we have $\Gamma_{i}=0$ for some $i$. By condition 2 , the total effective rate $R_{T}$ is bounded by

$$
R_{T}(\boldsymbol{\Gamma}) \leq \max _{j} \sum_{i=1, i \neq j}^{N} R_{i}
$$

given that $\boldsymbol{\Gamma}$ is a point at the boundary.

If $R_{T}\left(\boldsymbol{\Gamma}^{*}\right)$ is greater than the upper bound shown above, it possesses a global maximum at $\boldsymbol{\Gamma}^{*}$. If not, we let $\boldsymbol{\Gamma}^{\prime}=\boldsymbol{\Gamma}^{*}$ and keep $\boldsymbol{\Gamma}^{\prime}$ constant. Suppose now we increase $W$. The local maximum changes accordingly, and we still denote it by $\boldsymbol{\Gamma}^{*}$. Since $\Gamma_{i}^{\prime}$ is fixed and $x_{i}^{\prime}=\Gamma_{i}^{\prime} W / R_{i}, x_{i}^{\prime}$ will increase. When $W \rightarrow \infty$, we have $x_{i}^{\prime} \rightarrow \infty$. Thus, by condition $2, R_{T}\left(\boldsymbol{\Gamma}^{\prime}\right) \rightarrow$ $\sum_{i=1}^{N} R_{i}$. Since $R_{T}\left(\boldsymbol{\Gamma}^{*}\right)>R_{T}\left(\boldsymbol{\Gamma}^{\prime}\right), R_{T}$ attains a strong global maximum at $\boldsymbol{\Gamma}^{*}$.

A stationary harmonious solution does not necessarily yield a maximum. If $W$ is very small, the harmonious solution may

${ }^{1}$ A function $f$ attains a strong global maximum at $\mathbf{x}_{0}$ if $f\left(\mathbf{x}_{0}\right)>f(\mathbf{x})$ for all $\mathbf{x} \neq \mathbf{x}_{0}$ [9]. yield a global minimum. Thus, we have a dominated schedule. We state our result in Theorem 2. The proof is similar to that of Theorem 1 and can be found in [14].

Theorem 2: For the multirate power control problem with $N$ users, if the bandwidth is small enough, then the capacity optimization problem has a harmonious solution at which a strong global minimum can be attained.

\section{LARGE-SCALE SYSTEMS}

In this section, we consider two asymptotic cases. We first consider the case when the bandwidth is large. In the second case, the number of users and the bandwidth are both large. Our observations are summarized as follows.

Observation 1: If the bandwidth is large, then the optimal solution is close to the "proportional" solution

$$
\frac{\Gamma_{i}^{*}}{R_{i}} \approx c \quad \forall i
$$

where $c$ is a constant.

As mentioned in the proof of Theorem 1, when $W$ increases, the curve $k_{i}\left(x_{i}\right)$ will move down. Asymptotically, when $W \rightarrow$ $\infty, k_{i}\left(x_{i}\right) \rightarrow f^{\prime}\left(x_{i}\right)$ for all $i$. Hence, for large $W$, the stationary point can be obtained by solving

$$
W f^{\prime}\left(x_{i}^{*}\right)=\lambda
$$

In other words, $x_{i}^{*}$ is approximately constant for all $i$. It follows that $\Gamma_{i}^{*}$ is approximately proportional to $R_{i}$. Since for sufficiently large $W, x_{i}^{*}=\Gamma_{i}^{*} W / R_{i}>x_{0}$ for all $i$, the "proportional" solution yields a global maximum.

Observation 2: If the number of users and the bandwidth are both large, then the "proportional" solution yields a stationary point.

1) If $f^{\prime \prime}(x)<0$ for all $x$, it yields a global maximum.

2) If $\mathcal{P}\left(f^{\prime \prime}(x), \beta_{0}\right)$, it yields a global maximum provided that

$$
\frac{W}{\sum_{i=1}^{N} R_{i}} \gg \beta_{0}
$$

and it yields a global minimum provided that

$$
\frac{W}{\sum_{i=1}^{N} R_{i}} \ll \beta_{0} .
$$

In the above discussion, we have observed that the "proportional" solution yields a stationary point. This result is independent of $N$. However, if $N \rightarrow \infty$, we have $\Gamma_{i}^{*} \rightarrow 0$ for all $i$. Thus, $x_{i}^{*}$ is not necessarily greater than $x_{0}$. Hence, the point may not yield a maximum.

If $N$ is large, we have $Q_{T} \gg Q_{i}$. We further assume that $Q_{T} \gg \eta$. Then, we have the following approximation:

$$
\Gamma_{i}^{*} \approx \frac{Q_{i}^{*}}{Q_{T}}
$$


Hence, the "proportional" solution can be expressed as

$$
\frac{Q_{i}^{*}}{R_{i}} \approx c
$$

where $c$ is a constant.

Substituting it back to (39), we obtain

$$
\Gamma_{i}^{*} \approx \frac{R_{i}}{\sum_{j} R_{j}}
$$

Thus, we have

$$
x_{i}^{*}=\frac{\Gamma_{i}^{*}}{W} R_{i} \approx \frac{W}{\sum_{j} R_{j}} .
$$

If $f^{\prime \prime}(x)<0$ for all $x$, then when $W$ is large, $R_{T}$ becomes concave and $\mathrm{x}^{*}$ must yield a global maximum.

However, if we have $\mathcal{P}\left(f^{\prime \prime}(x), \beta_{0}\right), \mathrm{x}^{*}$ may not yield a maximum. When $W \rightarrow \infty$, by Lemma $3, \alpha_{i} \rightarrow \beta_{0}$ for all $i$. Hence, if $x_{i}^{*}>\beta_{0}$ for all $i$, a local maximum is attained at $\mathrm{x}^{*}$. Following the same line of reasoning as in the proof of Theorem 1, a global maximum is attained at $\mathrm{x}^{*}$ if

$$
\frac{W}{\sum_{i} R_{i}} \gg \beta_{0}
$$

Similarly, if we have $\mathcal{P}\left(f^{\prime \prime}(x), \beta_{0}\right)$, then a global minimum is attained at $\mathrm{x}^{*}$ if

$$
\frac{W}{\sum_{i} R_{i}} \ll \beta_{0} .
$$

In a large-scale system, our power control rule has a rather simple form. The SIR target of each mobile terminal can simply be set proportional to its transmission rate. This avoids a large amount of computations. Though this "proportional" solution makes it very simple to apply in practice, it is worth noting that some kinds of admission control is needed to ensure that the ratio $W / \sum_{j} R_{j}$ is large enough.

\section{QoS CONSIDERATION}

In our previous discussion, we derive the optimal SIR allocation scheme. We show that a globally optimal, harmonious solution exists provided that the bandwidth is large enough. A harmonious schedule assigns a strictly positive $\Gamma_{i}$ to mobile $i$. Thus, in principle, every mobile is allowed to transmit its data simultaneously. In practice, however, too small an SIR of a link renders communication impossible. It is necessary to guarantee a minimum rate for each mobile by imposing an SIR constraint

$$
\Gamma_{i} \geq \gamma_{i}
$$

for $i=1,2, \ldots, N$.
A necessary and sufficient condition for the feasibility of the problem is that

$$
\sum_{i=1}^{N} \frac{1}{1+\gamma_{i}} \leq K
$$

We assume that proper admission control is exercised such that (46) is satisfied. Then the following procedure can be applied to determine the optimal SIR vector.

1) Initialization: Let $L=K$ and $\mathcal{A}=\{1,2, \ldots, N\}$.

2) Compute $\Gamma_{i}^{*}$ for $i \in \mathcal{A}$ by solving the following equations:

$$
\begin{aligned}
\frac{\Gamma_{i}^{*}}{R_{i}} & =c, \quad \text { for all } i \in \mathcal{A} \\
\sum_{i \in \mathcal{A}} \frac{1}{1+\Gamma_{i}^{*}} & =L .
\end{aligned}
$$

3) If $\Gamma_{i}^{*} \geq \gamma_{i}$ for all $i$, exit.

4) For each $i \in \mathcal{A}$, if $\Gamma_{i}^{*}<\gamma_{i}$, let $\Gamma_{i}^{*}=\gamma_{i}$; let $L=L-\left(1 /\left(1+\gamma_{i}\right)\right)$; remove $i$ from $\mathcal{A}$.

\section{5) Go to 2.}

This procedure ensures that the minimum SIR requirement of each user is satisfied. If it is possible, some users will be assigned a higher SIR than is required. For those users, the assignment is essentially the "proportional solution."

\section{EXTENSION TO THE BASIC MODEL}

In our model, there is no delay constraint for the terminals. However, in a multimedia system, some terminals may require services which are delay-sensitive. For example, the traditional voice terminals cannot tolerate a large delay. Thus, it is appropriate to divide the multimedia terminals into two different classes. Class 1 terminals refer to those which can tolerate a large delay. Class 2 terminals refer to those which require real-time delivery. Our results in the previous sections are applicable to class 1 terminals. Now we extend our model to include the class 2 terminals. We will show that the problem can be decoupled and our previous results still hold.

We consider class 2 terminals first. We assume that each class 2 terminal has a QoS requirement which is given in terms of BER. It is well known that there is a strong relationship between the BER and the SIR. So we assume that the BER requirement can be translated into an SIR requirement. As a result, for class 2 terminals, we have the following constraints:

$$
\Gamma_{i}^{(2)} \geq \gamma_{i}^{(2)}, \quad i=1,2, \ldots, N^{(2)}
$$

where $\Gamma_{i}^{(2)}$ is the SIR of terminal $i$ and $\gamma_{i}^{(2)}$ is its SIR requirement. We use the superscript (2) to indicate that the quantity refers to a class 2 terminal. Without the superscript, the quantity refers to a class 1 terminal. 
Note that the SIR of class 2 terminal $i, \Gamma_{i}^{(2)}$, is given by

$$
\Gamma_{i}^{(2)}=\frac{Q_{i}^{(2)}}{\sum_{j \neq i} Q_{j}^{(2)}+Q_{T}+\eta}
$$

where $Q_{T}$, as before, is the total received power of class 1 terminals.

Our previous model remains unchanged except that the SIR of class 1 terminals is changed from (2) to the following:

$$
\Gamma_{i}=\frac{Q_{i}}{\sum_{j \neq i} Q_{j}+Q_{T}^{(2)}+\eta}
$$

where $Q_{T}^{(2)}$ is the total received power of class 2 terminals. Our objective is to find feasible received power vectors $\mathbf{Q}$ and $\mathbf{Q}^{(2)}$, such that the effective data rate of class 1 terminals $R_{T}$ is maximized, under the constraint given by (49).

We have the following observation about the optimal solution. The results are similar to that derived in [12]. In their work, only the class 2 traffic is considered. Our model, however, includes two classes of traffic.

Assume that at the optimal solution, the $i$ th constraint is a strict inequality

$$
\Gamma_{i}^{(2)}>\gamma_{i}
$$

Then we can decrease $Q_{i}^{(2)}$ without violating the constraint. As a result, the SIR of all class 1 terminals increase, which leads to an increase in the total effective data rate, $R_{T}$.

Therefore, at the optimal solution, the constraints given in (49) are all met with equality. It means that all the class 2 terminals sustains the minimum acceptable QoS. Thus, we have the following system of linear equations:

$$
\frac{Q_{i}^{(2)}}{\sum_{j \neq i} Q_{j}^{(2)}+Q_{T}+\eta}=\gamma_{i}^{(2)}, \quad \text { for } i=1,2, \ldots, N^{(2)} \text {. }
$$

By solving these equations, we obtain the optimal solution for class 2 terminals

$$
Q_{i}^{(2) *}=\frac{\gamma_{i}^{(2)}}{\gamma_{i}^{(2)}+1} \times \frac{Q_{T}+\eta}{1-\sum_{j=1}^{N^{(2)}} \frac{\gamma_{j}^{(2)}}{\gamma_{j}^{(2)}+1}}
$$

for $i=1,2, \ldots, N^{(2)}$.

We define the traffic load of class 2 terminal $i, \rho_{i}$, as follows:

$$
\rho_{i}=\frac{\gamma_{i}^{(2)}}{\gamma_{i}^{(2)}+1} .
$$

Let $\rho$ be the total traffic load of class 2 terminals

$$
\rho=\sum_{j=1}^{N^{(2)}} \rho_{i}
$$

Then we have

$$
Q_{i}^{(2) *}=\frac{\rho_{i}}{1-\rho}\left(Q_{T}+\eta\right)
$$

and

$$
Q_{T}^{(2) *}=\frac{\rho}{1-\rho}\left(Q_{T}+\eta\right)
$$

From the positivity of the power vector $\mathbf{Q}^{(2)}$ and (54), the following condition imposes a capacity constraint on the class 2 traffic:

$$
\rho<1 \text {. }
$$

If there is only one medium within class 2, i.e., $\gamma_{i}^{(2)}=\gamma_{j}^{(2)}$ for all $i$ and $j$, then the power control scheme for class 2 terminals reduces to the conventional constant received power method.

Now the optimal power vector of class 2 terminals has been determined. Substituting (58) into (51), we have

$$
\Gamma_{i}=\frac{Q_{i}}{\sum_{j \neq i} Q_{j}+\frac{\rho Q_{T}+\eta}{1-\rho}}
$$

Equation (60) has the same form as (2). Thus, our results derived in the previous sections remain the same. The existence of class 2 terminals has no effect on the power control strategy for class 1 terminals.

Consider the $E_{b} / J_{0}$ of the class 1 terminals

$$
\begin{aligned}
\left(\frac{E_{b}}{J_{0}}\right)_{i} & =\Gamma_{i} \times \frac{W}{R_{i}} \\
& =\frac{Q_{i}}{Q_{T}-Q_{i}+Q_{T}^{(2) *}+\eta} \times \frac{W}{R_{i}} \\
& =\frac{Q_{i}}{Q_{T}-Q_{i}+\frac{\rho}{1-\rho}\left(Q_{T}+\eta\right)+\eta} \times \frac{W}{R_{i}} \\
& =\frac{Q_{i}}{Q_{T}-(1-\rho) Q_{i}+\eta} \times \frac{(1-\rho) W}{R_{i}} .
\end{aligned}
$$

If the number of class 1 terminals, $N$, is large, then $Q_{T} \gg$ $(1-\rho) Q_{i}$. Thus, we have

$$
\left(\frac{E_{b}}{J_{0}}\right)_{i} \approx \frac{Q_{i}}{Q_{T}+\eta} \times \frac{(1-\rho) W}{R_{i}} .
$$

Note that the $E_{b} / J_{0}$ of each class 1 terminal in the system is approximately the same as another system which consists of solely class 1 terminals and has bandwidth $(1-\rho) W$. In other words, the existence of class 2 traffic in effect reduces the bandwidth for class 1 traffic by a factor of $(1-\rho)$.

\section{NUMERICAL STUDIES}

We have already shown that the problem consisting of two classes of traffic can be decoupled. Therefore, in this section, we consider only class 1 terminals.

We consider a single-cell system which has three active users. The channel is assumed to be a BSC. BPSK and DPSK will 
$W=0.5 R 1, R 1=64 \mathrm{kbps}, \mathrm{R} 2=64 \mathrm{kbps}, \mathrm{R} 3=64 \mathrm{kbps}$

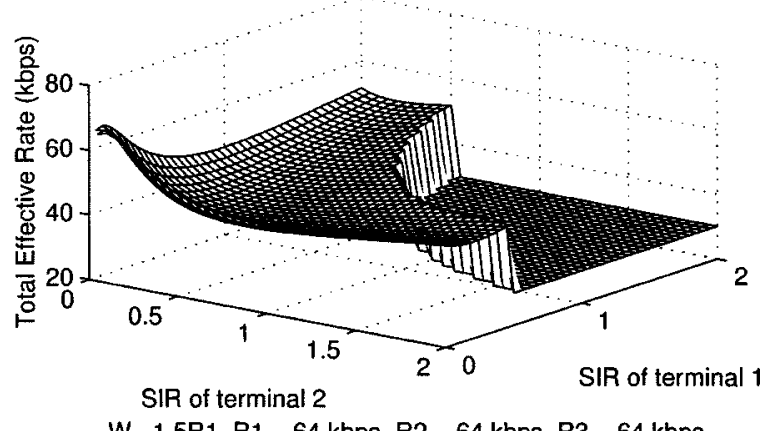

$\mathrm{W}=1.5 \mathrm{R} 1, \mathrm{R} 1=64 \mathrm{kbps}, \mathrm{R} 2=64 \mathrm{kbps}, \mathrm{R} 3=64 \mathrm{kbps}$

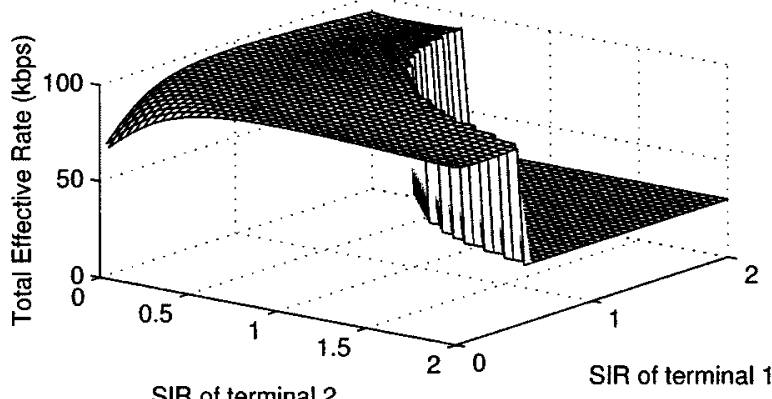

SIR of terminal 2

$W=4.5 R 1, R 1=64 \mathrm{kbps}, R 2=64 \mathrm{kbps}, \mathrm{R} 3=64 \mathrm{kbps}$

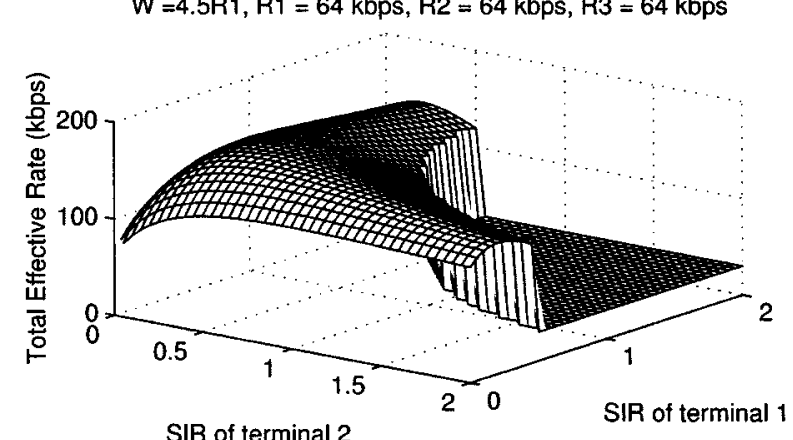

Fig. 3. BPSK modulation, $R_{i}=64 \mathrm{~kb} / \mathrm{s}$ for all $i, 3$ cases $(W=0.5 R, 1.5 R$ and $4.5 R$ ).

be used as the modulation schemes. Receiver noise is assumed negligible.

First of all, we assume that the three terminals transmit at the same rate $R$, where $R=64 \mathrm{~kb} / \mathrm{s}$. For BPSK modulation, we consider three cases: $W=0.5 R, 1.5 R$, and $3 R$. By symmetry, the stationary point must be located at $\Gamma_{i}=0.5$ for all $i$. From Fig. 3, it can be seen that $\boldsymbol{\Gamma}^{*}=(0.5,0.5,0.5)$ changes from a minimum to a maximum as $W$ increases. Since $\boldsymbol{\Gamma}^{*}$ is known in advance, by (75) and (76), it is easy to find that the extremum attained at $\boldsymbol{\Gamma}^{*}$ changes from a minimum to a maximum when $W / R$ exceeds 1.456 .

Similarly, we plot the result for DPSK in Fig. 4. We consider the cases where $W=1.5 R, 3 R$, and $6 R$. Again, the stationary point changes from a minimum to a maximum as $W$ increases. By (80) and (81), it can be found that the extremum attained at $\Gamma^{*}$ changes from a minimum to a maximum when $W / R$ exceeds 2.961 .

Next, we investigate the case where the three mobile terminals transmit at different rates. We assume that $R_{1}=64 \mathrm{~kb} / \mathrm{s}$, $R_{2}=32 \mathrm{~kb} / \mathrm{s}$, and $R_{3}=48 \mathrm{~kb} / \mathrm{s}$. $R_{T}$ possesses either a global
$W=1.5 R 1, R 1=64 \mathrm{kbps}, R 2=64 \mathrm{kbps}, \mathrm{R} 3=64 \mathrm{kbps}$

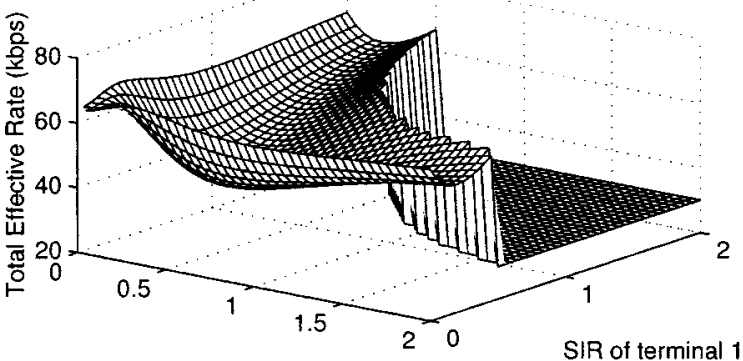

SIR of terminal 2

$\mathrm{W}=3 \mathrm{R} 1, \mathrm{R} 1=64 \mathrm{kbps}, \mathrm{R} 2=64 \mathrm{kbps}, \mathrm{R} 3=64 \mathrm{kbps}$

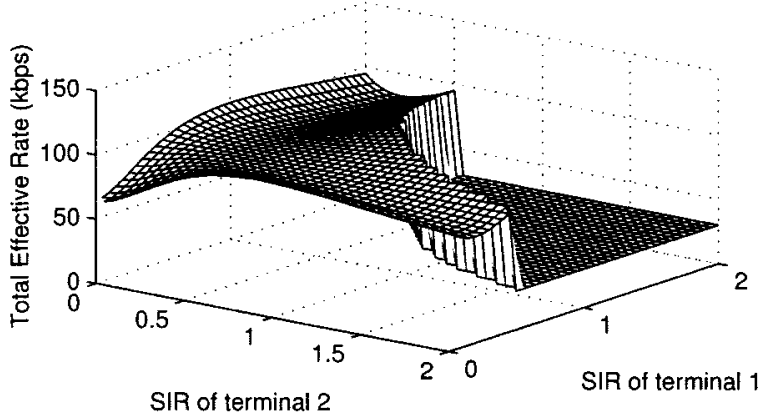

$\mathrm{W}=6 \mathrm{R} 1, \mathrm{R} 1=64 \mathrm{kbps}, \mathrm{R} 2=64 \mathrm{kbps}, \mathrm{R} 3=64 \mathrm{kbps}$

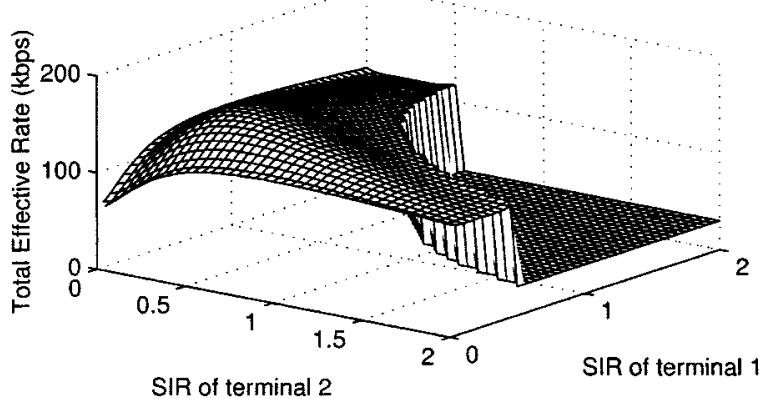

Fig. 4. DPSK modulation, $R_{i}=64 \mathrm{~kb} / \mathrm{s}$ for all $i, 3$ cases $(W=1.5 R, 3 R$ and $6 R$ ).

minimum or a global maximum depending whether $W$ is small or large. Graphs similar to Figs. 3 and 4 have been obtained and can be found in [14]. In Tables I and II, we show the ratio $\Gamma_{i}^{*} / R_{i}$ for different values of $W$ using BPSK and DPSK, respectively. When $W$ increases, the ratio for different users become closer and closer to each other. This result validates that in Observation 1.

Finally, we perform a numerical experiment to verify Observation 2 . We consider a system where there are 250 terminals. A BSC model with DPSK modulation is assumed. We let $10 \mathrm{~kb} / \mathrm{s}$ be the basic data rate. Except for terminal 1 and terminal 2, we assume that all the terminals transmit at the basic rate. Terminal 1 is assumed to transmit at a rate $R_{1}=4 \times$ basic rate $=40 \mathrm{~kb} / \mathrm{s}$. Terminal 2 is assumed to transmit at a rate $R_{2}=2 \times$ basic rate $=$ $20 \mathrm{~kb} / \mathrm{s}$. Since there are 250 users in the system, this is a 250-dimensional optimization problem. In this study, we investigate the effect of power control on the first two users only. The received power of all other users are normalized to one. Receiver noise is assumed negligible. The graphs showing the total effective rate against the received power of users 1 and 2 for $W=1$, 3 , and $5 \mathrm{Mb} / \mathrm{s}$ are plotted in Figs. 5-7, respectively. 
TABLE I

BPSK MOdULATION: RATIO $\Gamma^{*} / R_{i}$ FOR DIFFERENT VALUES OF $W$

\begin{tabular}{c|c|c|c|c}
\hline$W$ & $10^{5} \Gamma_{1}^{*} / R_{1}$ & $10^{5} \Gamma_{2}^{*} / R_{2}$ & $10^{5} \Gamma_{3}^{*} / R_{3}$ & Effective Rate (kbps) \\
\hline $5 R_{1}$ & 1.16 & 0.97 & 1.06 & 13.82 \\
\hline $10 R_{1}$ & 1.11 & 1.03 & 1.07 & 14.38 \\
\hline $15 R_{1}$ & 1.09 & 1.04 & 1.07 & 14.40 \\
\hline $20 R_{1}$ & 1.09 & 1.05 & 1.07 & 14.40 \\
\hline $40 R_{1}$ & 1.08 & 1.06 & 1.07 & 14.40 \\
\hline
\end{tabular}

TABLE II

DPSK MOdUlation: RATIO $\Gamma_{i}^{*} / R_{i}$ FOR DifFeRENT VALUES OF $W$

\begin{tabular}{c|c|c|c|c}
\hline$W$ & $10^{5} \Gamma_{1}^{*} / R_{1}$ & $10^{5} \Gamma_{2}^{*} / R_{2}$ & $10^{5} \Gamma_{3}^{*} / R_{3}$ & Effective Rate (kbps) \\
\hline $5 R_{1}$ & 1.19 & 0.94 & 1.06 & 12.71 \\
\hline $10 R_{1}$ & 1.11 & 1.02 & 1.07 & 14.31 \\
\hline $15 R_{1}$ & 1.09 & 1.04 & 1.07 & 14.40 \\
\hline $20 R_{1}$ & 1.09 & 1.05 & 1.07 & 14.40 \\
\hline $40 R_{1}$ & 1.08 & 1.06 & 1.07 & 14.40 \\
\hline
\end{tabular}

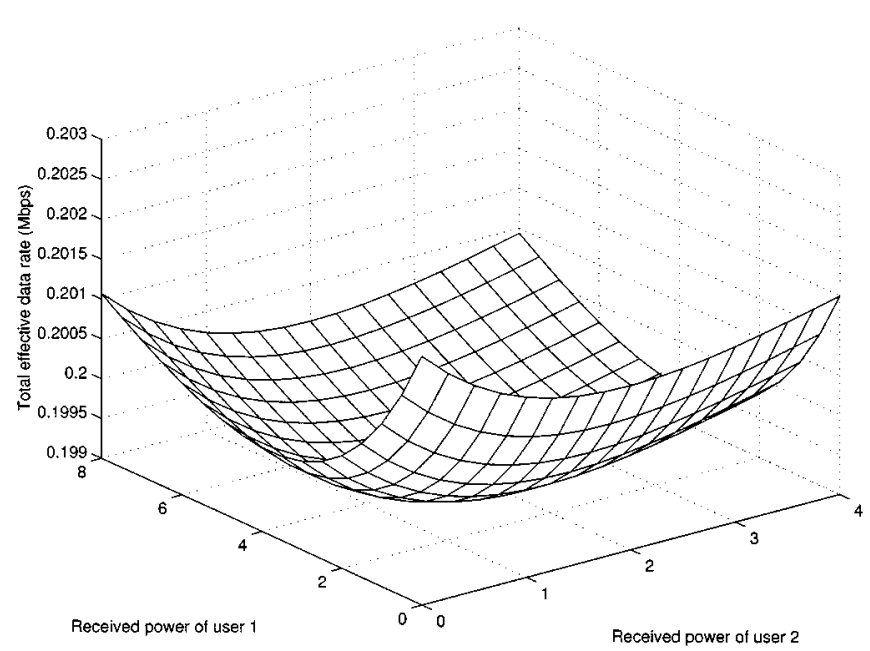

Fig. 5. Effect of power control on two users out of $250(W=1 \mathrm{Mb} / \mathrm{s})$.

For BSC model with DPSK modulation, $\beta_{0}=0.831$. According to Observation 2, the "proportional" solution yields a global maximum if

$$
\frac{W}{\sum_{i} R_{i}} \gg 0.831 .
$$

In this example, $\sum_{i} R_{i}=2.54 \mathrm{Mb} / \mathrm{s}$. Thus, the above condition becomes

$$
W \gg 2.11 \mathrm{MHz}
$$

Observation 2 also implies that the "proportional" solution yields a global minimum if

$$
W \ll 2.11 \mathrm{MHz} .
$$

In this example, the "proportional" solution $\left(Q_{1}^{*}, Q_{2}^{*}\right)$ is equal to $(4,2)$. It can be seen that this is a stationary point in all the three cases. In Fig. 5, $W$ is smaller than $2.11 \mathrm{MHz}$. The "proportional" solution yields a global minimum. In the second case, $W$ is equal to $3 \mathrm{MHz}$. It yields a local maximum but $W$

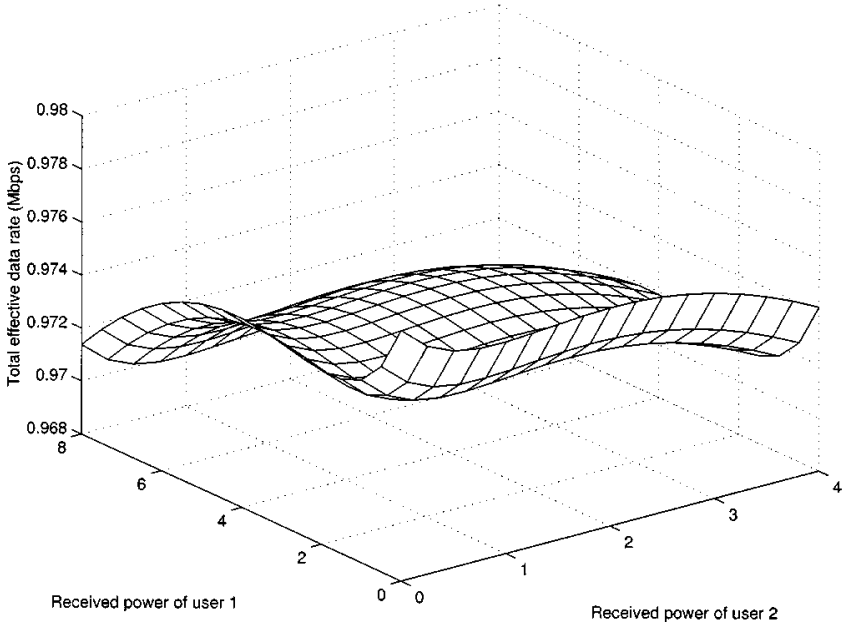

Fig. 6. Effect of power control on two users out of 250 ( $W=3 \mathrm{Mb} / \mathrm{s})$.

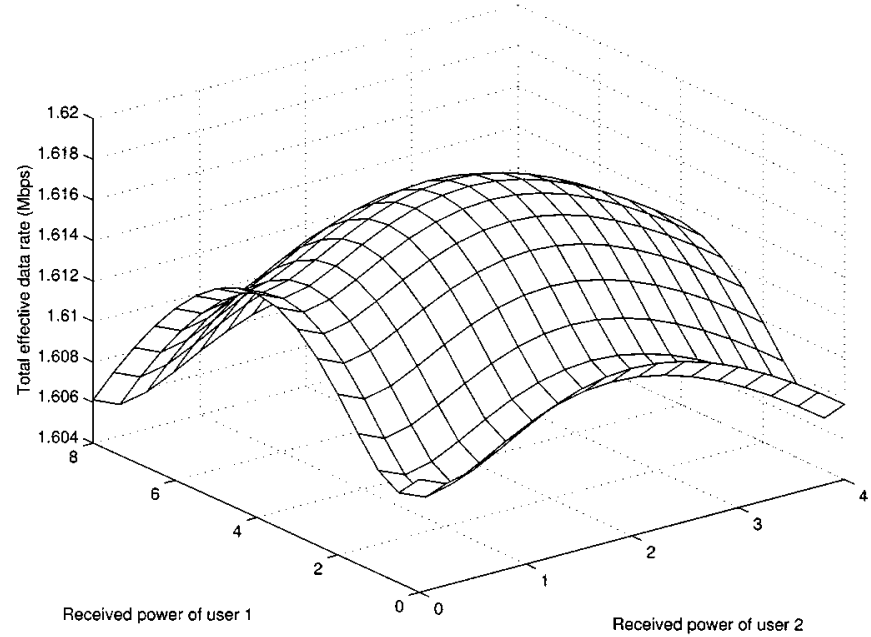

Fig. 7. Effect of power control on two users out of 250 ( $W=5 \mathrm{Mb} / \mathrm{s})$.

is not large enough and the global maximum is attained at the boundary. In the third case, $W$, being equal to $5 \mathrm{MHz}$, is large enough such that the solution yields a global maximum. Our result agrees with Observation 2.

The same experiment have been performed using BSC model with BPSK modulation. Since $f^{\prime \prime}(x)$ is always negative if BPSK is used, the "proportional" solution always yields a global maximum. For conciseness, we omit the graphs here.

\section{CONCLUSION}

In this paper, we have addressed issues in designing a multimedia CDMA network. In a multimedia system, different terminals have different QoS requirement and different resource constraints. For instance, data terminals and voice terminals require very different objective functions. To handle this problem, we divide them into two classes. One is delay-tolerable and the other is delay-sensitive. For delay-tolerable ones, we formulate the problem as a constrained optimization problem, with the objective of maximizing the total effective rate. An optimal power control strategy has been derived. When the bandwidth is large, the power control rule takes a very simple form, that is, the received SIR being proportional to the transmission rate. Hence, 
the rule can be easily put into practice. By specifying the target SIR, distributed power control algorithms such as that proposed in [15] can be used.

For a multimedia system which contains also delay-sensitive terminals, the nature of the problem remains unchanged. We show that the existence of the delay-sensitive terminals in effect reduces the bandwidth for the class of delay-tolerable terminals by a factor of $1-\rho$.

Our results are independent of the modulation/coding scheme and the channel characteristics. Thus, the power control strategy can be applied to many different situations. In our model, a single cell is considered and intercell interference is ignored. Future work may include extending the results to the multicell situation.

APPENDIX A

\section{DERIVATIVES OF $f(x)$ FOR BSC WITH BPSK AND DPSK MODULATION SCHEMES}

Assume a BSC. The $f(x)$ and its first two derivatives are given as follows:

$$
\begin{aligned}
f(x) & =1+p(x) \log (x)+(1-p(x)) \log (1-p(x)) \\
f^{\prime}(x) & =-\log \left(\frac{1-p(x)}{p(x)}\right) p^{\prime}(x) \\
f^{\prime \prime}(x) & =\log \left(\frac{1-p(x)}{p(x)}\right) p^{\prime \prime}(x)+\frac{\left(p^{\prime}(x)\right)^{2}}{p(x)(1-p(x)) \ln 2}
\end{aligned}
$$

where $p(x)$ is the BER.

For BPSK, we have the following results:

$$
\begin{aligned}
p(x)= & \frac{1}{2} \operatorname{erfc} \sqrt{x} \\
p^{\prime}(x)= & -\frac{1}{2} \sqrt{\frac{1}{\pi x}} e^{-x} \\
p^{\prime \prime}(x)= & -\left(\frac{1}{2 x}+1\right) p^{\prime}(x) \\
\frac{\partial L}{\partial \Gamma_{i}}= & \frac{1}{2} \sqrt{\frac{W R_{i}}{\pi \Gamma_{i}}} e^{-\Gamma_{i} W / R_{i}} \log \left(\frac{1-p\left(\frac{\Gamma_{i} W}{R_{i}}\right)}{p\left(\frac{\Gamma_{i} W}{R_{i}}\right)}\right) \\
\frac{\partial^{2} L}{\partial \Gamma_{i}^{2}}= & \frac{\lambda}{4 \pi \Gamma_{i} \ln 2} e^{-\Gamma_{i} W / R_{i}} \\
& \cdot\left[\frac{\Gamma^{2}}{p\left(\frac{\Gamma_{i} W}{R_{i}}\right)\left(1-p\left(\frac{\Gamma_{i} W}{R_{i}}\right)\right)}-\left(\frac{R_{i}}{\Gamma_{i} W}+2\right)\right. \\
& \left.\cdot \sqrt{\frac{\pi \Gamma_{i} W}{R_{i}}} \ln \left(\frac{1-p\left(\frac{\Gamma_{i} W}{R_{i}}\right)}{p\left(\frac{\Gamma_{i} W}{R_{i}}\right)}\right)\right]+\frac{2 \lambda}{\left(1+\Gamma_{i}\right)^{3}} .
\end{aligned}
$$

For DPSK, we have the following results:

$$
\begin{aligned}
p(x)= & \frac{1}{2} \exp (-x) \\
p^{\prime}(x)= & -\frac{1}{2} \exp (-x) \\
p^{\prime \prime}(x)= & \frac{1}{2} \exp (-x) \\
\frac{\partial L}{\partial \Gamma_{i}}= & \frac{W}{2} e^{-\Gamma_{i} W / R_{i}} \log \left(2 e^{\Gamma_{i} W / R_{i}}-1\right)-\frac{\lambda}{\left(1+\Gamma_{i}\right)^{2}} \\
\frac{\partial^{2} L}{\partial \Gamma_{i}^{2}}= & \frac{W^{2}}{2 R_{i} \ln 2} e^{-\Gamma_{i} W / R_{i}} \\
& \cdot\left[\frac{2 e^{\Gamma_{i} W / R_{i}}}{2 e^{\Gamma_{i} W / R_{i}}-1}-\ln \left(2 e^{\Gamma_{i} W / R_{i}}-1\right)\right]+\frac{2 \lambda}{\left(1+\Gamma_{i}\right)^{3}} .
\end{aligned}
$$

\section{APPENDIX B \\ ADDiTIONAL LEMMA}

Lemma 3: Let

$$
s(x)=2 f^{\prime}(x)+(x+a) f^{\prime \prime}(x)
$$

where $a>0$ is a constant.

Suppose $f^{\prime \prime}(x)<0$ for all $x$. For sufficiently large $a$, we have

$$
s(x)<0 \quad \forall x>0 .
$$

If $\mathcal{P}\left(f^{\prime \prime}(x), \beta_{0}\right)$, we have

$$
\mathcal{P}\left(s(x), \alpha_{0}\right)
$$

where $\alpha_{0} \in\left(\beta_{0}, x_{0}\right)$. Furthermore, $\alpha_{0} \rightarrow \beta_{0}$ when $a \rightarrow \infty$.

Proof: Let

$$
u(x)=2 f^{\prime}(x)+x f^{\prime \prime}(x)
$$

and

$$
v(x)=f^{\prime \prime}(x) .
$$

Note that we have

$$
s(x)=u(x)+a v(x)
$$

and by condition $8, \mathcal{P}\left(u(x), x_{0}\right)$.

By condition $3, f^{\prime}(x)$ is bounded. If $f^{\prime \prime}(x)<0$ for any $x>0$, it is obvious that for a sufficiently large $a, s(x)<0$ for any $x>0$.

Now we consider the alternative $\mathcal{P}\left(f^{\prime \prime}(x), \beta_{0}\right)$. It implies that $v\left(\beta_{0}\right)=0$. Since $f^{\prime \prime}\left(x_{0}\right)=-\left(2 f^{\prime}\left(x_{0}\right) / x_{0}\right)<0$, we must have $\beta_{0}<x_{0}$.

It is clear that

$$
s(x)>0 \quad \forall x \in\left(0, \beta_{0}\right)
$$

and

$$
s(x)<0 \quad \forall x \in\left(x_{0}, \infty\right) .
$$

It remains to determine the sign of $s(x)$ for $x \in\left[\beta_{0}, x_{0}\right]$. 
Note that $v^{\prime}\left(\beta_{0}\right)<0$. Let

$$
v^{\prime}\left(\beta_{0}\right)=-m_{1}
$$

where $m_{1}>0$.

Since $v^{\prime}(x)$ is continuous (condition 4), there exists $\delta \in\left(0, x_{0}-\beta_{0}\right)$ such that

$$
v^{\prime}(x)<-\frac{m_{1}}{2} \quad \forall x \in\left[\beta_{0}, \beta_{0}+\delta\right]
$$

Since $u^{\prime}(x)$ is continuous (condition 4), it must be bounded in a closed region. Hence, we have

$$
u^{\prime}(x)<m_{2} \quad \forall x \in\left[\beta_{0}, \beta_{0}+\delta\right]
$$

for some $m_{2}>0$.

Define $a_{1}, a_{2}$, and $a$ as follows:

$$
\begin{aligned}
a_{1} & =\frac{4 m_{2}}{m_{1}} \\
a_{2} & =\frac{2 \max _{x \in\left[\beta_{0}, \beta_{0}+\delta\right]} u(x)}{-v\left(\beta_{0}+\delta\right)} \\
a & =\max \left(a_{1}, a_{2}\right) .
\end{aligned}
$$

Note that all of them are positive.

Consider the value of $s\left(\beta_{0}\right)$.

$$
\begin{aligned}
s\left(\beta_{0}\right) & =u\left(\beta_{0}\right)+a v\left(\beta_{0}\right) \\
& =u\left(\beta_{0}\right) \\
& >0 .
\end{aligned}
$$

Next we consider $s\left(\beta_{0}+\delta\right)$.

$$
\begin{aligned}
s\left(\beta_{0}+\delta\right) & <u\left(\beta_{0}+\delta\right)+a_{2} v\left(\beta_{0}+\delta\right) \\
& <0
\end{aligned}
$$

where the first inequality follows from the fact that $v\left(\beta_{0}+\delta\right)<$ 0 and $a>a_{2}$.

For any $x \in\left[\beta_{0}, \beta_{0}+\delta\right]$, we have

$$
\begin{aligned}
s^{\prime}(x) & =u^{\prime}(x)+a v^{\prime}(x) \\
& <u^{\prime}(x)+a_{1} v^{\prime}(x) \\
& <m_{2}+\left(\frac{4 m_{2}}{m_{1}}\right)\left(-\frac{m_{1}}{2}\right) \\
& <0 .
\end{aligned}
$$

Therefore, $s(x)$ is strictly decreasing in $\left[\beta_{0}, \beta_{0}+\delta\right]$ and there exists $\alpha_{0} \in\left(\beta_{0}, x_{0}\right)$ such that

$$
s\left(\alpha_{0}\right)=0 .
$$

Moreover, for $x \in\left[\beta_{0}+\delta, x_{0}\right]$, if

$$
a>\frac{\max _{\beta_{0}+\delta \leq x \leq x_{0}} u(x)}{-\min _{\beta_{0}+\delta \leq x \leq x_{0}} v(x)}
$$

then $s(x)<0$.

Hence, we have proved $\mathcal{P}\left(s(x), \alpha_{0}\right)$, where $\alpha_{0} \in\left(\beta_{0}, x_{0}\right)$. When $a \rightarrow \infty$, we have $s(x) / a \rightarrow f^{\prime \prime}(x)$. Thus, $\alpha_{0} \rightarrow \beta_{0}$ when $W \rightarrow \infty$.

\section{REFERENCES}

[1] A. Baier, U. C. Fiebig, W. Granzow, W. Koch, P. Teder, and J. Thielecke, "Design study for a CDMA-based third-generation mobile radio system," IEEE J. Select. Areas Commun., vol. 12, pp. 733-743, May 1994.

[2] T. M. Cover and J. Thomas, Elements of Information Theory. New York: Wiley, 1991.

[3] G. J. Foschini and Z. Miljanic, "A simple distributed autonomous power control algorithm and its convergence," IEEE Trans. Veh. Technol., vol. 42, pp. 641-646, Nov. 1993.

[4] S. Hanly and D. Tse, "Multiaccess fading channels-Part II: Delay-limited capacities," IEEE Trans. Veh. Technol., vol. 44, pp. 2816-2831, Nov. 1998.

[5] C. L. I and K. K. Sabnani, "Variable spreading gain CDMA with adaptive control for integrated traffic in wireless networks," in Proc. IEEE VTC'95, 1995, pp. 794-798.

[6] W. C. Y. Lee, Mobile Communications Design Fundamentals. New York: Wiley, 1993.

[7] D. Mitra and J. A. Morrison, "A novel distributed power control algorithm for classes of service in cellular CDMA networks," unpublished private communication.

[8] T. Ottosson and A. Svensson, "multirate schemes in DS/CDMA systems," in Proc. IEEE VTC'95, 1995, pp. 1006-1010.

[9] M. J. Panik, Classical Optimization: Foundations and Extensions. Amsterdam, The Netherlands: North-Holland, 1976.

[10] J. G. Proakis, Digital Communications, second ed: McGraw Hill, 1989.

[11] S. S. Rao, Optimization Theory and Applications. New York: Wiley, 1979

[12] A. S. Sampath, P. S. Kumar, and J. M. Holtzman, "Power control and resource management for a multimedia CDMA wireless system," in Proc. IEEE PIMRC'95, 1995.

[13] A. Sampath and J. M. Holtzman, "Access control of data in integrated voice/data CDMA systems: Benefits and tradeoffs," IEEE J. Select. Areas Commun., vol. 15, pp. 1511-1526, Oct. 1997.

[14] C. W. Sung, "Access control in wireless multimedia systems," Ph.D dissertation, Dept. Inform. Eng., Chinese Univ. Hong Kong, June 1998.

[15] C. W. Sung and W. S. Wong, "A distributed fixed-step power control algorithm with quantization and active link quality protection," IEEE Trans. Veh. Technol., vol. 48, pp. 553-562, Mar. 1999.

[16] C. W. Sung, K. K. Leung, and W. S. Wong, "A quality-based fixed-step power control algorithm with adaptive target threshold," IEEE Trans Veh. Technol., vol. 49, pp. 1430-1439, July 2000.

[17] D. Tse and S. Hanly, "Multiaccess fading channels-Part I: Polymatroid structure, optimal resource allocation and throughput capacities," IEEE Trans. Inform. Theory, vol. 44, pp. 2796-2815, Nov. 1998.

[18] A. M. Viterbi and A. J. Viterbi, "Erlang capacity of a power controlled CDMA system," IEEE J. Select. Areas Commun., vol. 11, pp. 892-900, Aug. 1993.

[19] J. Wu and R. Kohno, "A wireless multimedia CDMA system based on transmission power control," IEEE J. Select. Areas Commun., vol. 14, pp. 683-691, May 1996.

[20] J. T.-H. Wu and E. Geraniotis, "Power control in multi-media CDMA networks," in Proc. IEEE VTC'95, 1995, pp. 789-793.

[21] R. D. Yates, "A framework for uplink power control in cellular radio systems," IEEE J. Select. Areas Commun., vol. 13, pp. 1341-1348, Sept. 1995.

[22] S. Yao and E. Geraniotis, "Optimal power control law for multi-media multi-rate CDMA systems," in Proc. IEEE VTC'96, 1996, pp. 392-396. 


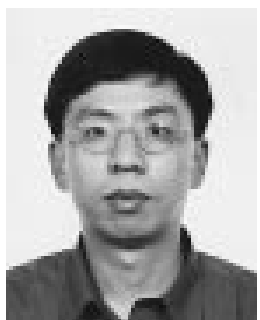

distributed control
Chi Wan Sung (M'98) received the B.Eng., M.Phil., and $\mathrm{Ph} . \mathrm{D}$. degrees in information engineering from the Chinese University of Hong Kong, Shatin, Hong Kong, in 1993, 1995, and 1998, respectively.

After graduation, he was appointed Visiting Assistant Professor with the Department of Information Engineering, Chinese University of Hong Kong, for one year. He is currently an Assistant Professor in the Department of Computer Science, City University of Hong Kong, Kowloon, Hong Kong. His research interests include wireless communication systems and

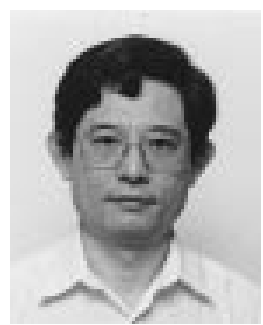

Wing Shing Wong (M'81-SM'90) received the combined M.A.B.A. degree (summa cum laude) from Yale University, New Haven, CT, in 1976. He received the M.S. and Ph.D. degrees from Harvard University, Cambridge, MA, in 1978 and 1980, respectively.

He joined AT\&T Bell Laboratories in 1982. From 1987 to 1992 , he managed a group of technical staff working on a number of research and development projects and consulting activities. He joined the Chinese University of Hong Kong in 1992 and is now a Professor of Information Engineering. He has been the Chairman of the Department of Information Engineering since 1995. He is actively involved ina a variety of research and development projects including topics such as mobile communication systems, search engine development, and information issues in estimation and control. He has published over 85 refereed journal and conference papers and is the recipient of multiple competitive research and development grants from the Hong Kong Research Grants Council and the Industry Support Fund. He leads the project on a chinese search engine, MoLi \& ANSeRS, which has been licensed to several high-tech companies.

Dr. Wong is a Fellow of the Hong Kong Institution of Engineers. He is a Discipline Representative for Information Engineering in HKIE and a Group Editor for the HKIE Transactions. He has been an Associate Editor of the IEEE TRANSACTIONS ON AUTOMATIC CONTROL for four years. He is the co-Editor-inChief of a new journal, Communications in Information and Systems. 